\section{Echisis}

OPINION

\title{
Chronic cough: from a complex dysfunction of the neurological circuit to the production of persistent cough
}

\author{
Adalberto Pacheco
}

\begin{abstract}
Correspondence to Dr Adalberto Pacheco, Chronic Cough Unit, Pneumology Service, Hospital Ramón y Cajal, Crtra. Colmenar Km 9, Madrid 28234, Spain; adalberto.pacheco@salud. madrid.org
\end{abstract}

Received 6 May 2014 Revised 26 May 2014 Accepted 31 May 2014 Published Online First 26 June 2014

\begin{abstract}
Chronic cough or cough that lasts more than 8 weeks, once a chest $\mathrm{x}$-ray and spirometry are confirmed normal, is caused by an alteration in a section of the route between peripheral receptors, mainly in the upper and lower airway and oesophagus, spinal cord and the cough centre in the brain stem involving the cortex. These mechanisms of cough have their homology in the circuit of chronic pain, and on that basis, should expand future research of chronic cough. Clinically chronic cough is easy to diagnose by an excessive response or hypertussia to low-intensity stimuli or banal stimuli, which we now call hypersensitivity cough syndrome, quantified by a positive reflex cough with capsaicin or citric acid. However, hypersensitivity cough syndrome can be impossible to quantify in the laboratory when the hyper-responsiveness originates in the central nervous system. This is normally caused by excessive peripheral input or convergence of stimuli from different sources. Once central hypersensitivity is acquired, peripheral input is not as important for activation of the cough.
\end{abstract}

The prevalence of chronic cough (CC) reported in studies in Europe and the USA varies between 9\% and 30\%. ${ }^{1}$ However, in two-thirds of patients the cough is completely or partially resistant to conventional treatment. ${ }^{2}$ In the last decade, the recognition of the similarities between the neurobiological mechanisms underpinning cough and pain has been an important development. ${ }^{3}$ To date, chronic pain has been studied in considerable depth, and now it is time to apply the findings from chronic pain studies to the management of CC.

The mechanism of cough is structured as a neurological circuit connecting the periphery to the central nervous system (CNS). In some instances the cerebral cortex is incorporated into the circuit in the form of the sensation of urge to cough, or voluntary cough, as opposed to the other kind of cough, the cough reflex. As in chronic pain, in CC the clinical problem arises when there is a prolonged imbalance between stimulus and response which impairs patients' quality of life and causes them to cough in response to mild stimuli, or even in response to innocuous stimuli such as talking or laughing. For this reason, this prolonged cough is now catalogued as a Cough Hypersensitivity Syndrome ${ }^{4}$ or sensory hyper-responsiveness to numerous mechanical or chemical stimuli occurring at different anatomical sites, especially the upper and lower airways and the oesophagus. These stimuli are transduced into electrical action potentials in the terminal fibres of the vagal or trigeminal neurons, all of which have their cell bodies in the spinal cord. These fibres, which are involved in the cough circuit, have been studied in depth in laboratory animals. Basically, there are three types ${ }^{5}$ : inflation and deflation receptors-rapidly adapting receptors and slowly adapting receptors-which do not play a major role in the

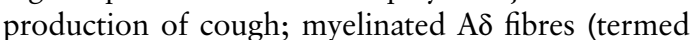
'cough receptors' by some authors) which respond to mechanical stimuli and acids; and unmyelinated C-fibres, which respond to thermal and chemical irritants such as capsaicin.

The first mechanism in the production of cough is the increased excitability of peripheral nerve endings to a given stimulus. Many types of chemical stimuli can elicit a neuroinflammatory response; these stimuli have been referred to collectively as an 'inflammatory soup'. ${ }^{3}$ The activation of TRPV1 and TRPA1 receptors present in C-fibres in the respiratory system has been explored in depth. One study in humans found that prostaglandin E2 inhalation increases the cough response to subsequently inhaled capsaicin, ${ }^{6}$ but the activation of A $\delta$ fibres may also be important; in a guinea pig model, after allergen-induced mast cell activation, a state of hypersensitivity to the mechanical stimuli that activate these nociceptors was observed. ${ }^{7}$

If the increase in excitability occurs in two different types of terminal fibres, this may lead to the phenomenon of convergence and synergy prior to cough. ${ }^{8}$ Confirmation of this kind of convergence was found when either bronchoconstriction (rapidly adapting receptor-mediated) or acid (C-fibre-mediated, in the distal third of the oesophagus) was provoked in guinea pigs; it was shown that neither event was sufficient to trigger cough alone, but simultaneous stimulation was able to cause coughing. ${ }^{9}$ In humans, oesophageal acid perfusion caused cough only in subjects with prior states of airway irritation such as asthma, but not in healthy subjects. ${ }^{10}$ This conjunction of two sites of activation has been identified by some authors as the origin of visceral hypersensitivity, a useful concept to explain that even normal levels of acid in the distal third of the oesophagus in healthy individuals may, in the case of concomitant CC, increase cough sensitivity. ${ }^{11}$ A similar convergence mechanism has been demonstrated in the nasal cavities and trachea to explain CC originating in the upper respiratory tract. ${ }^{12}$ These results suggest that action on two or more potential sources of cough 
Figure 1 Feedback neurological circuit of chronic cough.



such as the oesophagus and upper or lower airways may be beneficial for treatment of CC. This particular relationship was targeted in a clinical study of the connection between gastrooesophageal reflux and eosinophilic airway inflammation. ${ }^{13}$

The second mechanism involves more active participation on the part of the CNS, in the form of central sensitisation. Repeated peripheral stimuli can create a state of central hypersensitivity that may be reversible, although if the activity of nociceptors persists over time it may trigger a hypersensitivity known as spinal long-term potentiation. ${ }^{14}$ There are two interconnected ways in which this link between the periphery and the CNS can develop. One is via the interaction between terminal fibres, that is, when sensory input carried by the C-fibres to the cough centre (the nucleus tractus solitarius located in the brainstem) activate other nerve endings, whose sensitivity to these stimuli is probably increased by the synaptic plasticity in the cough circuit ${ }^{15}$; this has a synergistic effect, and produces cough. ${ }^{9}$ In one animal study, after stimulation of C-fibres at sites where there are no A $\delta$ fibres, such as the periphery of the lung or the oesophageal mucosa, an increase in input from A $\delta$ fibres in the mucosa of the larynx, trachea and bronchi was observed; then, the joint action of the A $\delta$ fibres and the C-fibres produced an increase in central sensitivity. ${ }^{8}$ In the context of chronic pain, an identical phenomenon occurs known as secondary hyperalge$\operatorname{sia}^{16}$; in cough, the condition is known as secondary hypertussivity, which refers to the way sensitisation originating in a specific somatic area is perceived as being more extended than it really is; for example, a peripheral stimulation of acid in the oesophagus might be perceived by the CNS as being caused by receptors at other sites such as the larynx or the airway.

The second potential influence of peripheral stimuli on the CNS is the effect exerted on the first neuron of the cough circuit by the microglia, a set of bone marrow derived cells that participate in primary immunity and are activated in chronic pain producing tumour necrosis factor $\alpha$ and interleukin- $1 b$; it may be that its modulation influences the degree of excitability in the spinal cord in response to cough stimuli. ${ }^{17} \mathrm{~A}$ last factor to bear in mind is the descending control on the nucleus tractus solitarius from the cerebral cortex (stimulatory and inhibitory), as in the pathophysiology of chronic pain; in turn, this control is influenced by the synapses in the pathway through the periaqueductal grey and the limbic brain, with the result that anxiety and depression may modulate the final efferent motor impulse. ${ }^{3}$
Sensitisation of the classic sites of the diagnostic triad of CC - the bronchi (eosinophilic or neutrophilic inflammation), the oesophagus (acid) and the upper airway-may then trigger sensitisation via the convergence and synergy in the CNS of sites where the presence of cough receptors is maximal, such as the larynx. Indeed, this could be an explanation for the common laryngeal and tracheal symptoms in patients with CC of all kinds, such as itching, tingling, stuck mucus, hoarseness, chest tightness, and so on, and the ease with which stimuli like strong smells and talking or singing trigger coughing. This state is termed hypertussivity (sensitisation to mild stimuli) or allotussivity (sensitisation to banal stimuli). So important are the laryngeal symptoms and triggers of CC that some authors have introduced the concept of laryngeal hypersensitivity in CC and regard it as fundamental to the clinical understanding of the condition; in fact, they apply it to several related problems such as vocal cord dysfunction, muscle tension dysphonia and even severe asthma. ${ }^{18}$ In studies of laryngeal hypersensitivity, multidisciplinary action including speech pathology treatments has managed to reduce C-fibre hypersensitivity to capsaicin, possibly by decreasing the excessive input from the larynx and consequently the hypersensitivity in the central area. ${ }^{19}$

In summary, peripheral and central hypersensitivity may be linked to each other in complex ways in the production of cough. The former may be the cause of cough, and the latter the consequence; alternatively, over time, central hypersensitivity may increase the excitability of different peripheral terminal fibres, as in a feedback loop; (figure 1) or both types of hypersensitivity may even develop independently to produce CC. Indeed, patients with chronic idiopathic cough have been found to have relatively normal sensitivities to challenge with tussive agents, yet develop allotussivity, ${ }^{20}$ a clinical manifestation inherent in central hypersensitivity. An important question is whether there are individuals, specially women more than men, ${ }^{21}$ with a genetic propensity to develop central sensitisation, and whether (as in the case of chronic pain) this encourages the development of CC, a state of hypersensitivity which complicates the problem even further, in terms of achieving the accurate diagnosis of the cause of the cough and with regards to applying appropriate treatment. This treatment may be based on decreasing the peripheral input, or on modifying the response of the CNS either with medication or through psychological interventions like education and mindfulness training. ${ }^{18} 22$ 


\section{Competing interests None.}

Provenance and peer review Not commissioned; externally peer reviewed.

\section{REFERENCES}

1 Chung KF, Pavord ID. Prevalence, pathogenesis, and causes of chronic cough. The Lancet 2008;371:1364-74.

2 Woodcock A, Young EC, Smith JA. New insights in cough. Br Med Bull 2010;96:61-73.

3 ÓNeill J, McMahon SB, Undem BJ. Chronic cough and pain: Janus faces in sensory neurobiology? Pulmonary Pharmacology $\alpha$ Therapeutics 2013;26:476-85.

4 Morice AH, Millqvist E, Belvisi MG, et al. Expert opinion on the Cough Hypersensitivity Syndrome in respiratory medicine. Eur Respir $J$ In press.

5 Canning BJ, Mazzone SB, Meeker SN, et al. Identification of the tracheal and laryngeal afferent neurons mediating cough in anaesthetized guinea-pigs. J Physiol 2004;557:543-58.

6 Coudry NB, Fuller RW, Pride NB. Sensitivity of the human cough reflex: effect of inflammatory mediators prostaglandin E2, bradykinin and histamine. Am Rev Respir Dis 1989;140:137-41.

7 Riccio MM, Myers AC, Undem BJ. Immunomodulation of afferent neurons in guinea-pig isolated airway. J Physiol 1996;491(Pt 2):499-509.

8 Mazzone SB, Mori N, Canning BJ. Synergistic interactions between airway afferent nerve subtypes regulating the cough reflex in guinea pigs. J Physiol 2005;569:559-73.

9 Canning BJ. Afferent nerves regulating the cough reflex: mechanisms and mediators of cough in disease. Otolaryngol Clin North Am 2010;43:15-25.

10 Wu DN, Yamauchi K, Kobayashi $H$, et al. Effects of esophageal acid perfusion on cough responsiveness in patients with bronchial asthma. Chest 2002;122:505-9.
11 Javorkova N, Varechova S, Pecova R, et al. Acidification of the oesophagus acutely increases the cough sensitivity in patients with gastro-oesophageal reflux and chronic cough. Neurogastroenterol Motil 2008;20:119-24.

12 Plevkova J, Antosiewicz J, Varechova S, et al. Convergence of nasal and tracheal neural pathways in modulating the cough response in guinea pigs. J Physiol Pharmacol 2009;60:89-93.

13 Pacheco A, Faro V, Cobeta I, et al. Gastro-oesophageal reflux, eosinophilic airway inflammation and chronic cough. Respirology 2011;16:994-9.

14 Sandkühler J, Gruber-Schoffnegger D. Hyperalgesia by synaptic long-term potentiation (LTP): an update. Curr Opin Pharmacol 2012;12:18-27.

15 Carr M, Undem B. Inflammation-induced plasticity of the afferent innervation of the airways plasticity. Environ Health Perspect 2001;109(Suppl 4):567-71.

16 Raja S, Campbell JN, Meyer RA. Evidence for different mechanisms of primary and secondary hyperalgesia following heart injury to the glabrous skin. Brain 1984;107:1179-88.

17 McMahon SB, Malcangio M. Current challenges in glia-pain biology. Neuron 2009;64:46-54.

18 Gibson PG, Simpson JL, Ryan NM, et al. Mechanisms of cough. Curr Opin Allergy Clin Immunol 2014;14:55-61.

19 Ryan NM, Vertigan A, Bone S, et al. Cough reflex sensitivity improves with speech language pathology management of refractory chronic persistent cough. Cough 2010;6:5

20 Morice $A H$, Fontana GA, Belvisi MG, et al. ERS guidelines on the assessment of cough. Eur Respir J 2007;29:1256-76.

21 Kelsall A, Decalmer S, McGuinness K, et al. Sex differences and predictors of objective cough frequency in chronic cough. Thorax 2009;64:393-8.

22 Young EC, Brammer $\mathrm{C}$, Owen $\mathrm{E}$, et al. The effect of mindfulness meditation on cough reflex sensitivity. Thorax 2009;64:993-8. 